Article

\title{
Using Automated On-Site Monitoring to Calibrate Empirical Models of Trihalomethanes Concentrations in Drinking Water
}

\author{
Thomas E. Watts, III ${ }^{1, \dagger}$, Robyn A. Snow ${ }^{1, \dagger}$, Aaron W. Brown ${ }^{1, \dagger}$, J. C. York ${ }^{2, \dagger}$, Greg Fantom ${ }^{2, \dagger}$, \\ Paul S. Simone, Jr. ${ }^{1,3, \dagger, *}$ and Gary L. Emmert ${ }^{1,3, \dagger, *}$
}

1 Department of Chemistry, The University of Memphis, Rm 213 Smith Chemistry Bldg., Memphis, TN 38152, USA; E-Mails: tewatts@memphis.edu (T.E.W.); rasnow@memphis.edu (R.A.S.); awbrown1@memphis.edu (A.W.B.)

2 The City of Lebanon, TN Water Treatment Plant, 7 Gilmore Hill Road, Lebanon, TN 37087, USA; E-Mails: yorkj@lebanontn.org (J.C.Y.); greg_fantom@yahoo.com (G.F.)

3 Foundation Instruments, Inc., Collierville, TN 38017, USA

$\dagger$ These authors contributed equally to this work.

* Authors to whom correspondence should be addressed; E-Mails: psimone@memphis.edu (P.S.S.); gemmert@memphis.edu (G.L.E.); G.L.E. Tel.: +1-901-678-2636 (P.S.S.); +1-901-678-3671 (G.L.E.); Fax: +1-901-678-3447 (P.S.S. \& G.L.E.).

Academic Editor: Abua Ikem

Received: 5 June 2015 / Accepted: 12 September 2015 / Published: 5 October 2015

\begin{abstract}
An automated, on-site trihalomethanes concentration data set from a conventional water treatment plant was used to optimize powdered activated carbon and pre-chlorination doses. The trihalomethanes concentration data set was used with commonly monitored water quality parameters to improve an empirical model of trihalomethanes formation. A calibrated model was used to predict trihalomethanes concentrations the following year. The agreement between the models and measurements was evaluated. The original model predicted trihalomethanes concentrations within $\sim 10 \mu \mathrm{g} \cdot \mathrm{L}^{-1}$ of the measurement. Calibration improved model prediction by a factor of three to five times better than the literature model.
\end{abstract}

Keywords: disinfection by-products; trihalomethanes; on-line monitoring; process control; empirical models 


\section{Introduction}

Compared to a century ago, the incidence of waterborne disease in the United States has become exceedingly rare. This is due in no small part to water chlorination which many consider the most significant public health development of the 20th century [1]. Unfortunately, despite all its benefits, water chlorination results in the formation of carcinogenic disinfection by-products (DBPs) from the reactions of free available chlorine with natural organic matter in source waters [1-5].

The trihalomethanes (THMs) are the most common class of DBPs. This group includes four chemical compounds, chloroform $\left(\mathrm{CHCl}_{3}\right)$, bromodichloromethane $\left(\mathrm{CHBrCl}_{2}\right)$, chlorodibromomethane $\left(\mathrm{CHBr}_{2} \mathrm{Cl}\right)$ and bromoform $\left(\mathrm{CHBr}_{3}\right)$. The total concentration of these four THMs (Total THMs) is regulated and should not exceed the maximum contaminant level (MCL) of $0.080 \mathrm{mg} \cdot \mathrm{L}^{-1}$ (or $80 \mu \mathrm{g} \cdot \mathrm{L}^{-1}$ ) [2,3] in finished drinking water. Data from the Environmental Working Group Drinking Water Database collected in 2007 [5] demonstrates that one in five legal violations in drinking water is a violation of the MCL for Total THMs. Clearly, many utilities struggle each year to meet these regulations and the occurrence of THMs formation in public drinking water is widespread.

Recently, these regulations have become more difficult to meet for water treatment plants (WTPs) across the country. Stage 1 of the DBP rule [2] has transitioned to the Stage 2 DBP rule which has been enforced since 2012 [3]. Under Stage 1 [2], WTPs reported a running annual average to establish compliance with the MCLs. Thus, Stage 1 allowed WTPs to sample from several different locations in their distribution system and average these results over the entire distribution system. This means that WTPs could establish compliance by averaging sites in their system that exhibited lower THMs concentrations with sites that exhibited higher concentrations of THMs to yield a running annual average less than the MCL. In the Stage 2 rule [3], the system-wide running annual average was replaced with the locational running annual average. The locational running annual average requires the WTPs to sample at specific sites (typically referred to as "hot spots") that exhibit the highest THMs concentrations. The locational running annual average is reported for each specific site, thereby eliminating the ability to average the THMs results across the distribution system. This makes achieving compliance of the DBP Rule more difficult for many WTPs across the country [3].

THMs formation is dependent upon several factors [4-12], perhaps chief among them the quality of source water available to the water treatment plant. Source water quality varies from plant to plant, and the approach that WTPs take to minimize THMs formation may vary greatly from location to location. Many WTPs are left on their own to determine exactly what treatment practices are most effective for their specific situations - situations that may change from hour to hour with causes such as rainfall, seasonal temperature inversion events [13], and perhaps other factors. To respond in real-time, WTPs have historically used surrogates to account for their THMs reduction practices. For example, specific absorbance at UV 254 (SUVA) and Total Organic Carbon (TOC) measurements have been used as "proxy" measurements of THMs concentrations. SUVA and TOC measurements can be done "on-site" at WTPs with subsequent data used to gain control of and minimize THMs formation [12,14,15]. Neither of these methods provides direct measurement of THMs species and unfortunately, as will be explored here, the surrogacy assumed in their use may not always be valid.

There are a number of ways to directly measure individual and Total THMs concentrations in drinking water, but most are not suitable for real-time THMs measurements [16]. The United States Environmental 
Protection Agency (USEPA) has provided several methods that work well for compliance monitoring with USEPA 551.1 [17], 502.2 [18] and 524.2 [19] being the most common. USEPA 551.1 [17] uses liquid-liquid extraction and gas chromatography with an electron capture detector to extract, separate, and quantify THMs, respectively. USEPA 502.2 [18] uses purge and trap gas chromatography with a photoionization detector connected in series with an electrolytic conductivity detector. USEPA 524.2 [19] uses purge and trap gas chromatography with the increased specificity of a mass spectrometer to accomplish the same goal. All three methods excel for compliance monitoring of THMs. However, they are an expensive capital investment for many WTPs, require analytical expertise typically beyond that of many WTP operators, and are not suited for real-time measurements of THMs concentrations.

As an alternative to on-site USEPA analysis, many WTPs outsource their THMs compliance monitoring to contract laboratories. Contract laboratories offer advantages such as eliminating the needs for expensive instrumentation and highly skilled analysts to operate the instruments. Further, the contract laboratory handles the data interpretation and analysis needed to demonstrate compliance with USEPA regulations. However, if daily process control is the goal, outsourcing samples to contract laboratories may be an expensive proposition resulting from a large number of samples and long sample turnaround times ranging from weeks down to days if expensive priority analysis is used. Such data are hardly useful to operators for responding in real-time to problems as they arise. Operators need an on-site "THMs meter" that enables them to monitor THMs concentrations in real-time, automatically, so they can respond in real time to THMs formation events as they arise.

In the last five years, commercial devices have appeared on the market for on-site THMs monitoring [13,20-24]. Perhaps the oldest alternative method is Hach's THM II Plus, a manual kit for Total THMs analysis [20]. The semi-automated Parker THM Analyzer [21] offers on-site measurements of individual THMs concentrations, but does not operate on-line and requires manual sample collection for analysis. Recently, Parker released the On-line THM Analyzer for on-line THM measurements. The Multisensor Systems MS2000 [22] is an on-line Total THM analyzer that couples headspace techniques with a sensor array for THMs detection. The Aqua Metrology Systems THM-100 [23] is a fully-automated, on-line Total THMs analysis system that uses a purge and trap technology followed by a chemical reaction with pyridine and base with spectrophotometric analysis.

Foundation Instruments, Collierville, TN, USA, offers the Trihalomethanes-Rapid Response system (THM-RR) which is arguably the most rigorously tested [13,24-27] commercial THM system on the market. Side-by-side comparisons to USEPA Method 524.2 of individual and Total THMs concentrations demonstrated agreement between the two methods within $2 \mu \mathrm{g} \cdot \mathrm{L}^{-1}$ [13]. Speciation of THMs is essential when evaluating certain mitigation strategies such as aeration which preferentially removes chloroform and bromodichloromethane due to higher volatility compared to dibromochloromethane and bromoform.

The THM-RR system was used to establish an on-site monitoring program at the Lebanon, TN WTP where operational and environmental factors that influence THMs formation and mitigation were explored $[13,25]$. The THM-RR has proven to be a rugged and robust device. However, the key to success for developing an automated, on-site THMs monitoring program is to establish the process map detailing the baseline behavior of THMs at the WTP. Then with the process map in place, effective process optimization studies can be conducted to determine the best THMs control strategies for the WTP. Both the process map and process optimization studies are discussed in more detail in Section 3.1. 
Most of the work related to understanding the concentrations of THMs that occur in drinking water has focused on modeling their formation. THMs modeling using common water quality parameters has been favored over routine THMs measurements because the latter was more difficult and expensive, until recent availability of commercial THMs analyzers [13,20-25]. This was especially true in the early days of the THMs problem [28]. One of the first known models for THMs formation was developed by Minear and Morrow [29] followed by more than one hundred models created to predict DBP formation in drinking water [30]. The Minear and Morrow work [29] was followed by a model proposed by Amy et al. [31] that became the seminal THMs, haloacetic acids, chloral hydrate and bromate ion models [32] for drinking water.

The Amy model [32] was developed by building a database of source and coagulated water quality parameters from multiple water sources across the United States. These parameters included dissolved organic carbon (DOC), chlorine dose (D), bromide ion concentration $\left(\mathrm{Br}^{-}\right)$, water temperature (T), contact time $(t)$, and $\mathrm{pH}$ [32]. The models were constructed using both linear and non-linear regression of the water quality parameters in conjunction with measured THMs concentrations. The result was a statistical model that could predict individual and Total THMs concentrations. Empirical models provide WTPs the opportunity to estimate Total THMs concentrations using routinely measured parameters. However, the predictive accuracy of these models may depend on source water selection. Further, the reliability of the experimental measurements used to determine the empirical relationships [32] is critical. In essence, each WTP has unique source water chemistry and parameters that makes developing an accurate universal model difficult.

In this paper, the Amy model [32] is applied using the equation from the literature to generate Total THMs concentrations at the Lebanon, TN WTP using their routinely measured water quality parameters of finished drinking water. The only difference was that the bromide ion term was ignored since the source contained negligible bromide ion concentrations nor were brominated THMs prominent. Then, the model is calibrated with Total THMs concentrations determined using the THM-RR system at the Lebanon, TN WTP. The result is a model built on a large set of synchronized data consisting of the on-site and on-line Total THMs measurements and the routine water quality parameters necessary for the model. This large dataset, 136 sets of data points, was then used to predict Total THMs concentrations for the same time period of the following year. The Total THMs concentrations generated by the literature model and calibrated model are rigorously compared using Bland-Altman analysis to Total THMs concentrations measured directly by the THM-RR system. Here is reported for the first time a WTP with the ability to tailor their empirical model to be site specific in a cost-effective manner.

\section{Experimental Section}

\subsection{City of Lebanon, TN WTP}

The Lebanon, TN WTP is a 12 million gallons per day conventional WTP located next to the Cumberland River in Wilson County, Tennessee. Water is drawn from the Cumberland River and powdered activated carbon (PAC) (Norit Hydrodarco O) is added to control taste and odor and reduce DBP precursors. The water enters a mixing tank where a polyaluminum chloride coagulant is added before transferring to flocculation chambers. The water passes through tube settlers before being filtered 
through dual media gravity filters. A small pre-dose of gaseous chlorine is added on top of the filters to control bacteriological growth in the filter media. The filtered water is disinfected with gaseous chlorine followed by addition of blended phosphates as a corrosion inhibitor. After the required contact time, the finished drinking water is pumped to sanitized reservoirs, water towers, and to the customers' taps. The utility maintains an active laboratory where common drinking water parameters are measured and logged with each shift. The utility also has an active flushing program in place aimed at THMs control.

\subsection{The THM-Rapid Response System}

All THM measurements were made using the THM-RR system (Foundation Instruments, Inc., Collierville, TN, USA), operating on an hourly sampling schedule. The THM-RR is fully-automated from the start of sampling to reporting individual and Total THMs concentrations. The patented THM-RR system [13,24,25] (Figure 1) is a rugged and robust version of an earlier device [26,27].

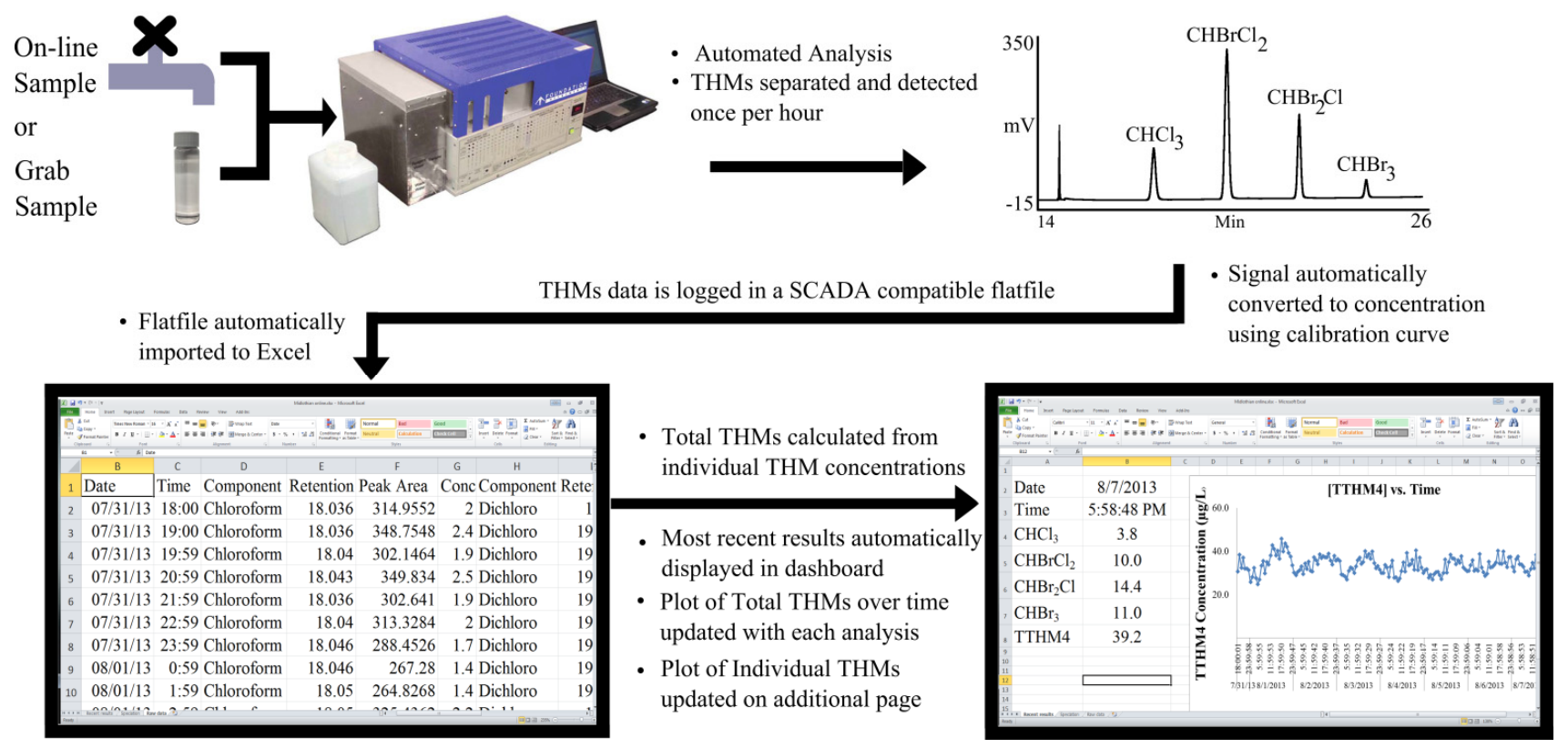

Figure 1. Illustration of operation of the Trihalomethanes-Rapid Response (THM-RR) system used to establish the program for automated on-line monitoring of individual and Total THMs concentrations in Lebanon, TN water treatment plant (WTP).

The operation of the THM-RR system is illustrated in Figure 1. Drinking water is sampled directly into the THM-RR from either a continuously flowing tap or a grab sample. The THM-RR uses a capillary membrane sampling device to allow pervaporation of the THMs into a nitrogen gas stream followed by introduction into a gas chromatograph for separation and detection [13,24-27]. The analytical signal for each THMs species is computer integrated followed by automated reporting and logging of THMs concentrations into a spreadsheet via external calibration curve. Finally, the THMs data set is displayed on the controlling PC in numeric and graphical format.

With an internet connection, the device may be remotely accessed with some control options available. The THM-RR sampling rate is one sample per hour with THMs results from the analysis typically 
available in $30 \mathrm{~min}$. The device can be switched between off-line grab sample and check standard analysis mode and on-line analysis using the installed, manual stream selection valve.

\subsection{Reagents and Standards}

Reagent water for standard preparation was distilled on-site at the Lebanon, TN WTP. The resulting distilled water does not contain any measurable organic or inorganic contaminants. The $0.2 \mathrm{mg} \cdot \mathrm{mL}^{-1}$ each THMs stock standard was purchased from AccuStandard (New Haven, CT, USA). A $4000 \mu \mathrm{g} \cdot \mathrm{L}^{-1}$ primary dilution standard was prepared volumetrically by diluting the stock standard into $50.00 \mathrm{~mL}$ of methanol. An $800 \mu \mathrm{g} \cdot \mathrm{L}^{-1}$ each THMs working standard was prepared using a $10 \mathrm{~mL} / 50 \mathrm{~mL}$ volumetric dilution in distilled water. Subsequently, all THMs calibration and check standards were prepared by diluting an appropriate volume of the working standard in $50.00 \mathrm{~mL}$ of distilled water. All preparation and analysis of the calibration and check standards was completed using three, $50.00 \mathrm{~mL}$ volumetric flasks, a $10 \mathrm{~mL}$ volumetric pipette and a $2 \mathrm{~mL}$ Mohr pipette with $0.1 \mathrm{~mL}$ increments. The typical concentrations for the calibration standards for each THM species are 1.6, 4.8, 8.0, 16, 32, $48 \mu \mathrm{g} \cdot \mathrm{L}^{-1}$. The check standard concentration is $3.2 \mu \mathrm{g} \cdot \mathrm{L}^{-1}$ for each THM species.

\subsection{Calibration, Linearity, Method Detection Limits, Accuracy and Precision}

The THM-RR is calibrated using a five point calibration curve. The range of this calibration curve is user adjustable to their specific needs, but typically ranges between $1.6 \mu \mathrm{g} \cdot \mathrm{L}^{-1}$ to $32 \mu \mathrm{g} \cdot \mathrm{L}^{-1}$ for each THMs species and from $6 \mu \mathrm{g} \cdot \mathrm{L}^{-1}$ to $128 \mu \mathrm{g} \cdot \mathrm{L}^{-1}$ for Total THMs. An optional standard extends the calibration range up to $48 \mu \mathrm{g} \cdot \mathrm{L}^{-1}$ for each THMs species and $192 \mu \mathrm{g} \cdot \mathrm{L}^{-1}$ for Total THMs. Automatically calculated least squares fits typically exhibit correlation coefficients ( $r$ values) greater than 0.99 over the calibration ranges for individual THMs species described above. Very low level $\left(\sim 10 \mathrm{ng} \cdot \mathrm{L}^{-1}\right)$ measurements have also been bracketed with similar results. The method detection limits (MDLs) for each individual THMs species (meaning the lowest concentrations of THMs that can be distinguished from noise) [33,34] range from 0.010 to $0.040 \mu \mathrm{g} \cdot \mathrm{L}^{-1}$ (10 to 40 parts per trillion) [13], or put another way, a factor of 2000 times below MCL values. The limits of quantitation [35], range from 0.03 to $0.1 \mu \mathrm{g} \cdot \mathrm{L}^{-1}[13]$. The accuracy (how close the measured value is to the true value) expressed as the mean percent recovery [36] on a check standard analyzed seven consecutive times is typically $80 \%$ at $0.3 \mu \mathrm{g} \cdot \mathrm{L}^{-1}$ and $99 \%$ at $20 \mu \mathrm{g} \cdot \mathrm{L}^{-1}$ [13]. The precision (the amount of scatter in the data) measured as the percent relative standard deviation (\% RSD) [36] of the check standard is $\pm 3 \%$ [13].

\subsection{On-Line Monitoring Using the THM-RR System}

The THM-RR is unique in that it comes standard with the ability to do on-line monitoring or easy switchover to analyze individual calibration and check standards or grab samples. For on-line sampling, the standard sampling loop consists of a length of Tygon ${ }^{\circledR}$ connected directly to a water tap and arranged into a loop with the exit going to drain. A length of polymer tubing is inserted through the side of the Tygon ${ }^{\circledR}$ tube and connected directly to the instrument. This configuration is standard for all THM-RR systems. During each hour of analysis, the THM-RR automatically samples from the drinking water stream for $15 \mathrm{~min}$ and then rinses the sampling device with distilled-deionized water for the remaining 
45 min to minimize carryover effects. The calibration is readily confirmed through analysis of a "check standard" and has been demonstrated to provide acceptable results for process control for months at a time [13]. The check standard is prepared in the same manner as a calibration standard, but is not used in generating the calibration data for the instrument. The frequency of check standard analysis can be determined by the user's needs. The THM-RR operates automatically for more than three weeks on a typical tank of relatively inexpensive Grade 5.0 nitrogen.

\section{Results and Discussion}

\subsection{Establishing an Automated, On-Site THMs Monitoring Program at the Lebanon, TN WTP}

The THM-RR system was installed at the Lebanon, TN WTP in April of 2013 to monitor THMs concentrations. Over the first week, no changes were made to the routine operating parameters of the plant to construct the "process map" (Figure 2A) [13,25]. The process map was constructed by monitoring THMs concentrations hourly, identifying patterns, and then determining their origin from within the WTP. Once the "normal" behavior was mapped, it was possible to make operational changes and accurately determine the effects of those changes [13,25]. Without knowing the process map, optimization studies may or may not make sense, depending on how sampling procedures are timed within the process map.

The Lebanon, TN WTP established a process map of their individual and Total THMs concentrations and determined that there were significant variations in the concentrations that occurred on hourly timescales $[13,25]$. Several of these short term events were correlated with water treatment processes and were shown to occur regularly during normal operations. With the process map in place, process optimization could be used with confidence that the changes measured were a result of the process changes and not from routine operations. During process optimization, the THM-RR system carefully monitored individual and Total THMs concentrations while doses of powdered activated carbon (PAC) and prechlorination were varied (Figure $2 \mathrm{~A}$ ).

PAC dosage was optimized first. During the process mapping phase (19-26 April 2013), THMs concentrations were monitored while the PAC concentration was held constant at $10 \mathrm{mg} \cdot \mathrm{L}^{-1}$. Between 27 April and 3 May, PAC concentration was increased to $15 \mathrm{mg} \cdot \mathrm{L}^{-1}$. In the final week of the PAC study (4-9 May), the dose was increased to $20 \mathrm{mg} \cdot \mathrm{L}^{-1}$ PAC. With each increase in PAC dose, the TOC concentrations of the finished water decreased (Figure 3). However, the concentrations of Total THMs demonstrated no visual decrease in concentration (Figure 2A). When plotted $v$ s. PAC dose, the observed Total THMs concentrations increased (Figure 3). It is generally accepted that as the TOC concentration decreases in the finished water, the concentrations of THMs will too, as more of their precursors are removed. Interestingly enough, this was not the case at the Lebanon, TN WTP during this period; instead, the opposite trend was observed. Apparently, the TOC that was being removed was not TOC that was being transformed into THM species. 


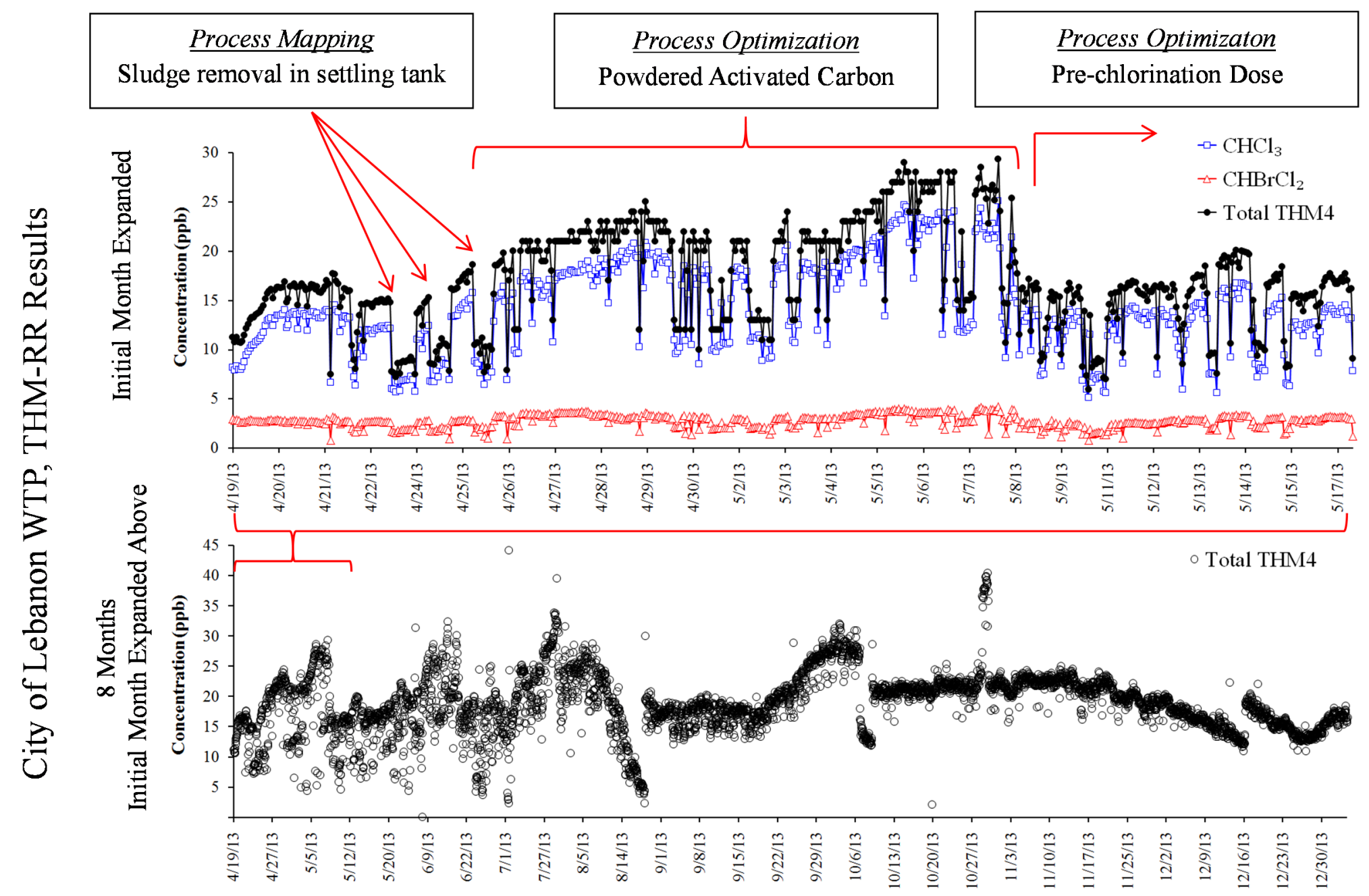

Figure 2. (A) Initial Month Expanded: Typical process map and two factor process optimization study conducted at the Lebanon, TN WTP for optimization of powdered activated carbon (PAC) dose and prechlorination dose; (B) The 8 month plot illustrates the large quantity of THMs concentration data obtained after establishing the on-line monitoring program at the Lebanon, TN WTP, and the consistent treatment practices implemented after 1 September 2013 to reduce THMs variability. 


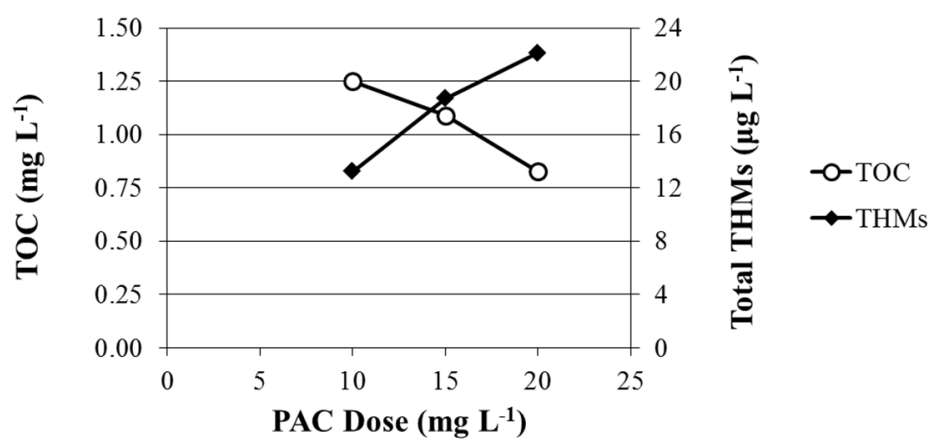

Figure 3. A plot of the powdered activated carbon optimization study; demonstrating a decrease in Total Organic Carbon (TOC) with increasing PAC dose and a simultaneous increase in Total THMs concentrations.

With these results in mind, PAC concentrations were reduced to an optimal concentration of $10 \mathrm{mg} \cdot \mathrm{L}^{-1}$ for controlling Total THMs concentrations. A simple economic study was done to compare the PAC use in 2013 after implementation of the monitoring program to the comparable period in 2012. Over this short time period, the WTP used 15,300 pounds less PAC in 2013, translating to a savings of $\$ 14,400$. Over the period of about one year, the Lebanon, TN WTP has paid for their THM-RR system in PAC savings alone.

In addition to the cost savings on PAC use, other operational practices have been streamlined at the Lebanon, TN WTP to minimize THMs concentrations and improve consistency. In a follow-on study, the pre-chlorination dose on top of the filters was also investigated. On 9 May and 10 May (Figure 2A), the concentration of chlorine dose at the pre-chlorination site was minimized guided by the THM-RR data so that the concentration of Total THMs was held to less than $20 \mu \mathrm{g} \cdot \mathrm{L}^{-1}$ and this control has continued to this day.

Finally, consider the overall effect on THMs concentrations when different WTP operators employ slightly different practices from shift to shift. At the Lebanon, TN WTP, three shifts are worked by different WTP operators. These WTP operators differ in experience and sometimes, personal preference, as to how they operate the WTP. The process maps created using THM-RR data identified trends traced back to differences in chlorine dose between the three operators. This difference in chlorine doses leads to shifts in THMs concentration of more than $10 \mu \mathrm{g} \cdot \mathrm{L}^{-1}$ on a day's timescale and $30 \mu \mathrm{g} \cdot \mathrm{L}^{-1}$ on a week's timescale. Once the trend was confirmed, the superintendent of the Lebanon, TN WTP developed standard chlorine dose protocols for all operators which have reduced shifts in THMs concentrations to less than $5 \mu \mathrm{g} \cdot \mathrm{L}^{-1}$ on a daily timescale [13] (Figure $2 \mathrm{~B}$ ).

The end results of these studies are exemplified in the lower plot of Figure $2 \mathrm{~B}$ by considering the left side of the plot compared to the right side of the plot. On the left side of the plot (before 1 September 2013), the overview is a utility with large variability in its treatment practices thus causing high variations in THMs concentrations. Prior to the on-site THMs monitoring, the causes of the variability were practically unknown. The right side of Figure 2B (after 1 September 2013) exemplifies the same WTP after having an on-site THMs monitoring program in place. On the right side, the superintendent has taken control of the WTP processes, optimized treatment practices, saved money, and improved water quality. Concurrently to this, the short- and long-term variability of Total THMs concentrations was radically reduced. 


\subsection{Calibrating Empirical Models in the Lebanon, TN WTP}

The recent availability of commercial on-site THM analyzers [13,21-25] has made it possible for WTP operators to obtain hours' timescale turnaround on their THMs concentrations. While the instruments are commercially available, their use has not been widely adopted. There are approximately 300 WTPs that have some sort of on-site program in use. Thus, empirical modeling is still a widespread practice. In this paper, the Amy model [32] is modified to mirror the water quality data that is collected at the Lebanon, TN WTP. While this results in a "re-calibration" of the Amy model, it should in no way detract from the creative, clever and robust work as originally reported [31,32] or indicate that their work was "wrong" in anyway. The detailed Amy empirical model as it appears in the literature is shown below (Equation (1)).

$$
\text { Total THMs }\left(\mu \mathrm{g} \cdot \mathrm{L}^{-1}\right)=0.0412(\mathrm{DOC})^{1.10}(\mathrm{D})^{0.152}\left(\mathrm{Br}^{-}\right)^{0.068}(\mathrm{~T})^{0.61}(\mathrm{pH})^{1.60}(t)^{0.260}
$$

The Amy literature model used raw water parameters whereas the work in this paper used finished water parameters. Instead of DOC, the work presented here used total organic carbon (TOC). At the WTP, the TOC concentrations and temperature are determined once per day (at 7:00 a.m.). The pH of the finished water is determined every four hours. The $\mathrm{pH}$ value used in the models corresponds to the 7:00 a.m. readings of TOC and temperature. The chlorine dose and contact time are each a daily average. The contact time depended upon the flow of water through the plant and is calculated by multiplying the free available chlorine at the point of entry by the time in minutes that it takes the drinking water to travel from the chlorination point to the point of entry. The Total THMs concentrations reported by the THM-RR each day at the 7:00 a.m. sampling time were used for calibration and comparison of the model; however the THM-RR reported THMs concentrations once per hour. Finally, the bromide ion concentration $\left(\mathrm{Br}^{-}\right)$term was ignored since the WTP's source water did not contain $\mathrm{Br}^{-}$ at appreciable concentrations.

The Amy literature model for THMs was based upon DOC concentrations that ranged from 1.2 to $10.6 \mathrm{mg} \cdot \mathrm{L}^{-1}$, chlorine doses ranging from 1.51 to $33.55 \mathrm{mg} \cdot \mathrm{L}^{-1}$, temperatures of $15-25{ }^{\circ} \mathrm{C}$, $\mathrm{pH}$ of $6.5-8.5$, and contact times ranging from 2 to $168 \mathrm{~h}$ [32]. During the calibration time period for the model, the water quality parameters at the Lebanon, TN WTP ranged as follows: TOC was $0.106-1.65 \mathrm{mg} \cdot \mathrm{L}^{-1}$; chlorine dose was $3.0-5.1 \mathrm{mg} \cdot \mathrm{L}^{-1}$; temperature was $8.5-26{ }^{\circ} \mathrm{C}$; $\mathrm{pH}$ was $7-7.7$; and contact time was 45-96 min. During the prediction period for the models, the water quality parameters at the Lebanon, TN WTP ranged as follows: TOC was $1.1-1.92 \mathrm{mg} \cdot \mathrm{L}^{-1}$; chlorine dose was $3.0-5.1 \mathrm{mg} \cdot \mathrm{L}^{-1}$; temperature was $10.5-26.5^{\circ} \mathrm{C}$; $\mathrm{pH}$ was $7-7.5$; and contact time was $30-81 \mathrm{~min}$. The concentrations of Total THMs for the Amy literature model development were as high as $800 \mu \mathrm{g} \cdot \mathrm{L}^{-1}$. In the work presented here, the Total THMs ranged from 9 to $55 \mu \mathrm{g} \cdot \mathrm{L}^{-1}$.

Initially, the Amy model was applied directly as it appeared in the literature (Equation (1)) with the exception of using the parameters as noted above. Total THMs concentrations were generated for an eight month period between May 2013 and January 2014 based on the measured water quality parameters. This data is plotted as a function of time (Amy Lit.) in Figure 4, along with the Total THMs concentrations measured by the THM-RR system during the same time period (Figure 4, "THM-RR"). Visually, the two methods show differences, but this is not surprising since the Amy model is based on source waters that did not include Tennessee. 


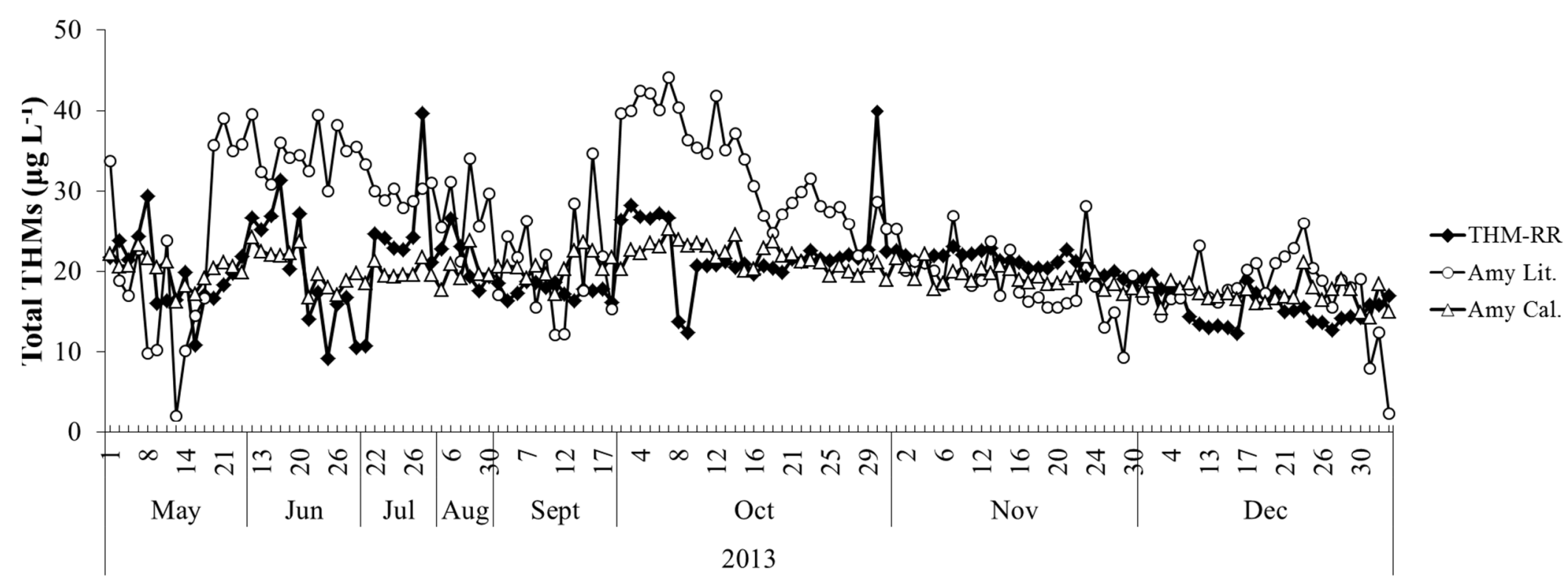

Time (Days)

Figure 4. A plot of Total THMs vs. time from 1 May 2013 through 3 January 2014 is shown. The plot includes Total THMs concentrations measured using the THM-RR, Total THMs concentrations calculated using the Amy Literature model (Amy Lit.), and Total THMs concentrations calculated after the Amy model has been calibrated (Amy Cal.). 
The Amy model [32] was then fitted using non-linear least squares analysis [9,37,38] to the Total THMs concentration data from the THM-RR system. This was accomplished by allowing the first coefficient and exponents of the water quality parameters to be variable during the fitting process and thus produce a "calibrated" model equation (Equation (2)). The calibrated, site specific model was then used to calculate THMs concentrations based on the measured water quality parameters which is plotted in Figure 4 (Amy Cal.). This was done to check the validity of the fitting process and determine whether the calibrated model generates THMs concentrations and trends similar to those of the THMs concentrations measured by the THM-RR. Visually, the Total THMs concentration data generated by the calibrated model appears to more closely follow the Total THMs concentration measurements from the THM-RR system. Visual evaluation is important, but can be misleading. The human eye can see apparent patterns where none exist and miss relationships that may be important.

$$
\text { Total THMs }\left(\mu \mathrm{g} \mathrm{L}^{-1}\right)=16.173(\mathrm{TOC})^{1.31}(\mathrm{D})^{0.917}(\mathrm{~T})^{0.112}(\mathrm{pH})^{-0.014}(t)^{-0.315}
$$

\subsection{Evaluating the Agreement between THM-RR Measurements and Empirical Models}

It is important to have a more quantitative approach than visual methods for evaluating agreement. The agreement between the Total THMs concentrations predicted by the empirical models and the Total THMs concentration measurements of the THM-RR can be compared using Bland-Altman analysis [39-42]. Bland-Altman analysis provides a statistical measurement of agreement between two methods and a tool to investigate whether systematic errors exist, such as concentration dependent bias. The agreement is evaluated by calculating the bias between the two methods for each set of instantaneous Total THMs concentrations. The use of any method in comparison studies as a "gold standard" is problematic [39]; nevertheless in this work for convention the "true value" is assumed to be the THM-RR measurement. Thus, the analytical bias [35] was calculated by taking the difference between the two measurements (Equation (3)).

$$
\text { Analytical Bias }\left(\mu \mathrm{g} \cdot \mathrm{L}^{-1}\right)=\text { Empirical model }\left(\mu \mathrm{g} \cdot \mathrm{L}^{-1}\right)-\mathrm{THM}-\mathrm{RR}\left(\mu \mathrm{g} \cdot \mathrm{L}^{-1}\right)
$$

Even though this convention is applied, the Bland-Altman analysis was done properly by plotting the analytical bias as defined in Equation (3) vs. the average of the Total THMs concentrations from the THM-RR and empirical model rather than just the THM-RR measurement [39]. The Bland-Altman analysis was done using Sigma Plot [22,39]. In general, the smaller the bias, the better the agreement of the results predicted by the two methods.

There were 136 direct comparisons made over eight months between the Amy Literature model and THM-RR system at the Lebanon, TN WTP. The THM-RR measurements exhibited concentration ranges of 9.2 to $39.9 \mu \mathrm{g} \cdot \mathrm{L}^{-1}$. These concentrations are well within the range encountered by a "typical" WTP that expect to observe Total THMs concentrations between $\sim 1$ and $100 \mu \mathrm{g} \cdot \mathrm{L}^{-1}$. These measurements were made with an instrument capable of detecting Total THMs concentrations of $0.05 \mu \mathrm{g} \cdot \mathrm{L}^{-1}$ with mean $\%$ recoveries in this concentration range of $97 \%$ to $103 \%$ with $2 \%$ RSD [13]. The literature model predicted Total THMs concentrations ranging from 2.0 to $44.1 \mu \mathrm{g} \cdot \mathrm{L}^{-1}$ while the calibrated model Total THMs concentrations ranged from 14.3 to $25.3 \mu \mathrm{g} \cdot \mathrm{L}^{-1}$, which are in good agreement with the THM-RR.

The Bland-Altman bias for the literature model was $4.7 \pm 8.9 \mu \mathrm{g} \cdot \mathrm{L}^{-1}$, with a $95 \%$ Confidence Interval (CI) of 3.2 to $6.1 \cdot \mu \mathrm{L}^{-1}$. When one considers that the model is being applied directly from the literature 
(Equation (1)) [32] outside of a water system in which it was developed, this is an incredibly high degree of agreement and thus a robust model. After calibration using the on-site THM-RR data, the Bland-Altman bias is reduced to $0.0 \pm 4.2 \mu \mathrm{g} \cdot \mathrm{L}^{-1}$ with $95 \% \mathrm{CI}$ ranging from -0.71 to $0.71 \mu \mathrm{g} \cdot \mathrm{L}^{-1}$. The degree of agreement is much improved by calibration, less than a single part-per-billion, and the relative error in the bias decreased by over $50 \%$. The analytical bias, as described earlier, gave virtually identical results to the Bland-Altman bias. The analytical bias is a simpler calculation and can be determined using a spreadsheet. The improved agreement after calibration of the model shows promise for this approach to generating site specific models.

The mean $\%$ recovery, a measure of the accuracy of the literature model, was $128 \% \pm 55 \%$. This is consistent with the bias measurements, indicating the literature model over-predicts compared to the THM-RR measurements. Unfortunately, the standard deviation of this measure indicates a high degree of variability in the accuracy of the model over time. Ideally, assuming the two methods agree exactly, one would expect mean $\%$ recoveries of $100 \%$. With the calibrated model, the mean $\%$ recovery values improve to $105 \% \pm 24 \%$ demonstrating improvement in accuracy and a decrease in relative error by greater than $50 \%$.

In Figure 5, four plots are presented related to the Bland-Altman bias calculations for calibration of the models. In plots A and B (Figure 5), a typical correlation plot for the concentrations of the literature model and calibrated model are plotted $v$. the THM-RR measurements, respectively. Visually, the two plots would appear to show some degree of correlation, though the calibrated model shows a better correlation to the THM-RR measurements over the literature model. However, this is to be expected as both methods were developed to measure and/or predict the same value. The problem with these plots is that the data will always cluster around a regression line (as shown in Figure 5) whatever the level of agreement. Even though this type of plot is commonly used, this may not be the best way to evaluate this type of data. Most of the plot is empty space. Additionally, as the range of measurements increases, the degree of agreement will appear to increase as well [39].

Instead, it is preferable to plot the difference between the two methods vs. their average [39], as shown in Figure 5C for the literature model and Figure 5D for the calibrated model. Such a plot makes it much easier to estimate the level of disagreement, identify outliers, and to identify any potential trends. The difference plots are similar to a residuals plot that follows model fitting and as such any observed patterns may be similarly identified. For example, in the literature model (Figure 5C), the bias becomes more positive as average Total THMs concentrations increase, whereas the calibrated model (Figure 5D) shows a more negative bias as average Total THMs concentrations increase. However, neither of these trends is particularly pronounced, and with the exception of the slight trends, the errors seem to be randomly distributed around the mean. Thus, apparently neither model suffers from strong systematic bias.

One shortcoming of calculating the bias, either with Bland-Altman analysis [39-42] or the analytical bias calculation [35] is that positive and negative differences may cancel one another out. This might result in bias values that are close to zero indicating an apparent agreement where it may not exist. The mean unsigned error (MUE) determines the absolute difference between any two pairs of measurements providing a different estimate of agreement [43]. The MUE of the model is calculated by taking the absolute value of each bias and calculating the average bias, thus minimizing the potential "canceling" effect. The MUE for the Amy literature model was $7.8 \pm 6.2 \mu \mathrm{g} \cdot \mathrm{L}^{-1}$ while the MUE for the calibrated, site specific model was $3.1 \pm 2.8 \mu \mathrm{g} \cdot \mathrm{L}^{-1}$. Hence, the Amy literature model predicted THMs 
concentrations that differed by $7.8 \mu \mathrm{g} \cdot \mathrm{L}^{-1}$ on average, positive or negative, while the calibrated model differed by $3.1 \mu \mathrm{g} \cdot \mathrm{L}^{-1}$ on average. For the literature model, the average difference between the MUE and Bland-Altman bias was $3.1 \mu \mathrm{g} \cdot \mathrm{L}^{-1}$. The difference for the calibrated model was identical. This indicates the magnitude of the cancellation effect is $\sim 3 \mu \mathrm{g} \cdot \mathrm{L}^{-1}$, which is within the error of the bias and thus acceptable.
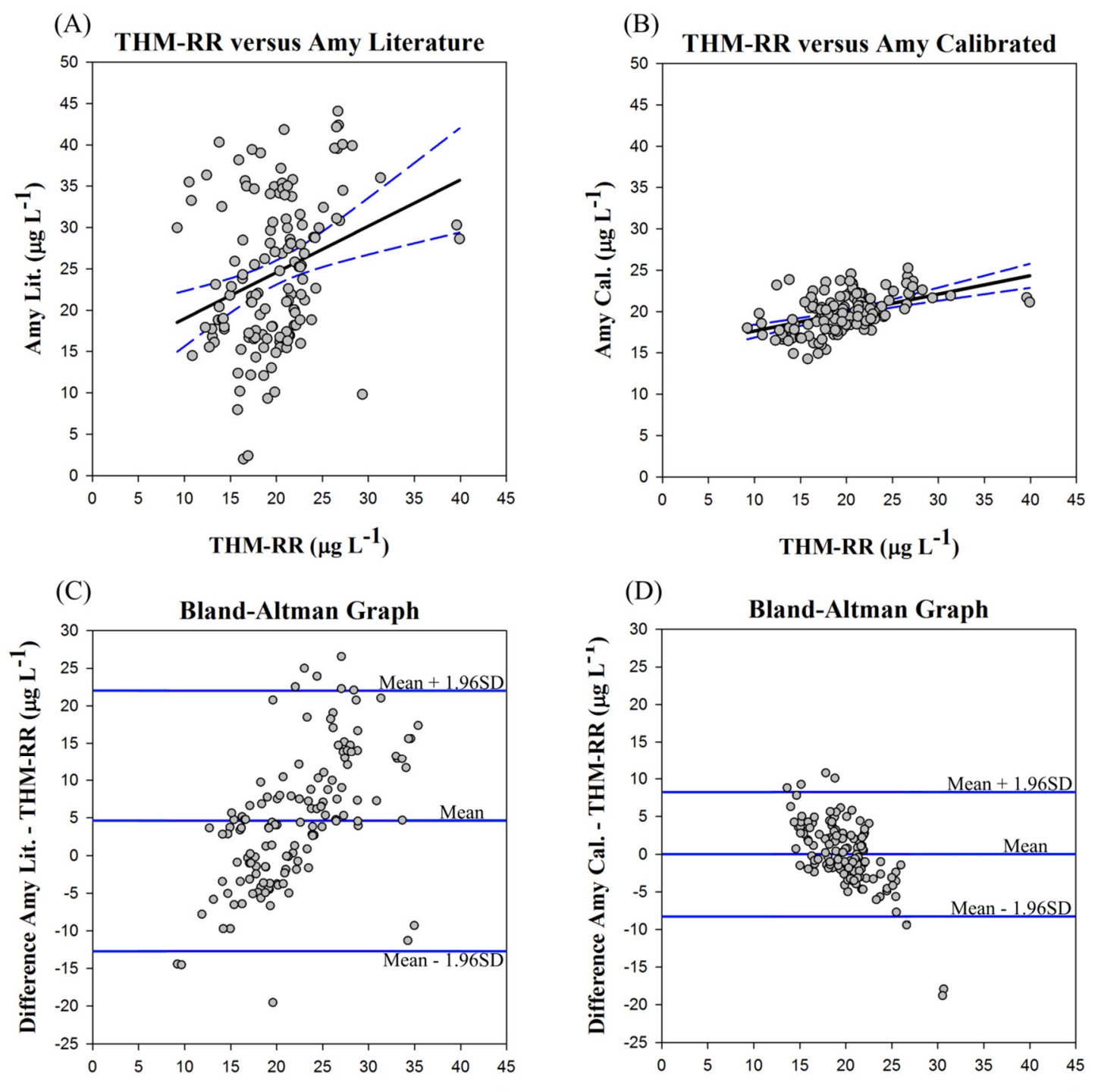

Average of THM-RR and Amy Lit. $\left(\mu \mathrm{g} \mathrm{L}^{-1}\right)$

Average of THM-RR and Amy Cal. $\left(\mu \mathrm{g} \mathrm{L}^{-1}\right)$

Figure 5. Bland-Altman Plots for the Amy literature and calibrated models for the period of 1 May 2013 through 3 January 2014. (A) Amy Literature generated Total THMs concentrations vs. THM-RR measurements; (B) Amy Calibrated generated Total THMs concentrations vs. THM-RR measurements; (C) Difference plot for the Amy Literature model and THM-RR measurements of Total THMs; (D) Difference plot for the Amy Calibrated model and THM-RR measurements of Total THMs.

When Total THMs concentrations are predicted in a time series as in process monitoring measurements, it is important that the predicted values from the two methods agree in time or closely "track well." The term "analyte tracking" is used to refer to the instantaneous behavior of two particular 
methods used simultaneously to measure the same species [13,27]. If there is a change in Total THMs concentrations, one would expect two methods to respond accordingly. Thus, if two adjacent samples at a particular point in time for each method are analyzed, the two methods should be responding the same way; i.e., one would expect that both methods (THM-RR and appropriate empirical model) should ideally be registering the same "instantaneous change"-either both positive, both negative, or both unchanged. Analyte tracking is thus a measurement of how well the THM-RR measurement and the empirical model prediction agree over time [13,27].

Analyte tracking was evaluated quantitatively by calculating the instantaneous concentration change for two adjacent samples at a particular point in time for each method. At each point in time, the difference in concentration at sampling time T1 and sampling time T2 (where T2 is greater than T1 and the two times are in consecutive order in the study) was calculated by subtracting the concentration of analyte at $\mathrm{T} 1$ from the concentration of analyte at $\mathrm{T} 2$. The two methods agree if both exhibit positive, negative or no change in concentration. The two methods will agree $33 \%$ of the time by random chance. The Total THMs concentration predicted by the Amy literature model tracked with the Total THMs measured by the THM-RR 52\% of the time and tracking for the calibrated model was $51 \%$. The differences in tracking between the literature and calibrated model are unlikely to be significant.

\subsection{Using Calibrated Empirical Models for Total THMs Predictions at the Lebanon, TN WTP}

The THM-RR provides an avenue to calibrate the models using high quality and quantities of THMs concentration data in a cost-effective manner. However, the ultimate use of these models, whether calibrated or not, is prediction of THMs concentrations at the WTP. In Section 3.2, the Amy literature model was used to predict Total THMs concentrations, evaluated, and subsequently re-fit using THMs concentrations data collected over an eight month period from 1 May 2013 through 2 January 2014. In this section, the ability of the Amy literature [32] and calibrated models to predict Total THMs concentrations in the following year (June-December 2014) are evaluated. The 2014 predictions are compared to THM-RR measurements using the same metrics (analytical bias [35], Bland-Altman bias [39-42] and MUE [43]) as described in the calibration process in the previous section. To be clear, no re-fitting or calibration was conducted for these comparisons. The comparison is based on using the Amy literature and calibrated models as predictive tools (Figure 6) and the THM-RR measurements of Total THMs concentrations at each point in time. 


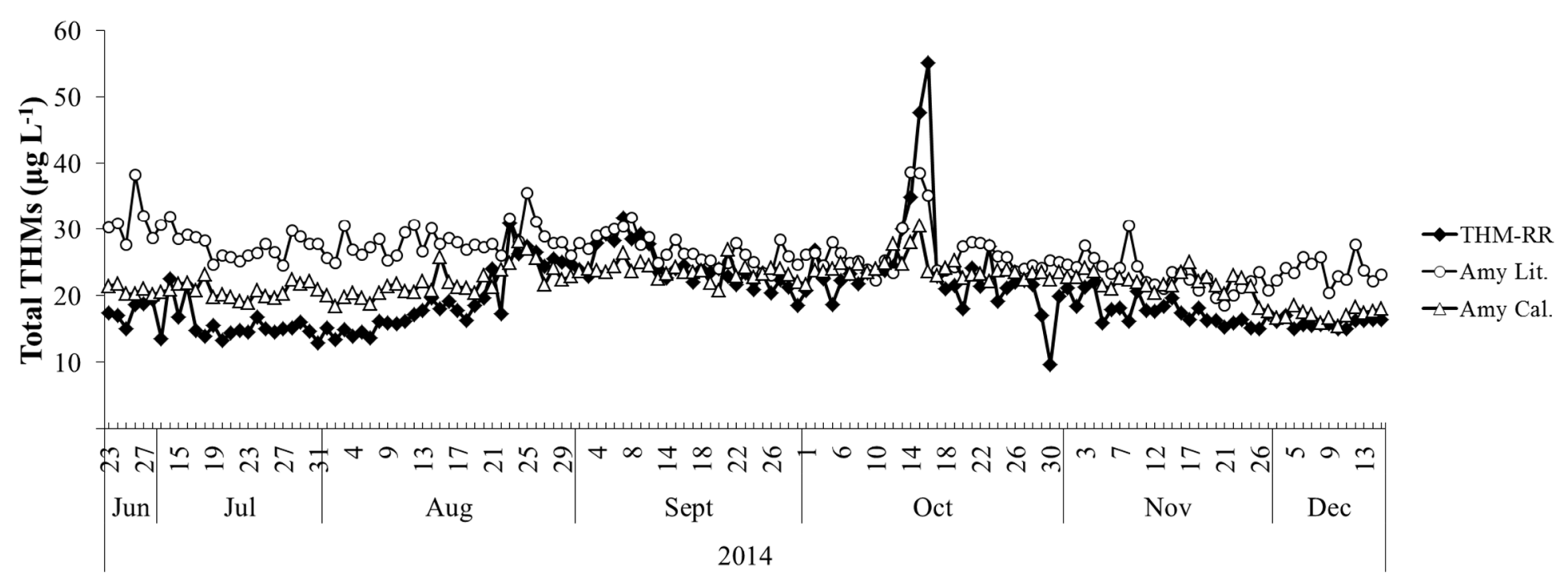

\section{Time (Days)}

Figure 6. A plot of Total THMs vs. time from 23 June 2014 through 16 December 2014 is shown where predicted Total THMs concentrations using the Amy Literature model (Amy Lit.) and Amy Calibrated model (Amy Cal.) are compared to the Total THMs measurements from the THM-RR. 
There were 147 direct comparisons made during 23 June 2014 to 16 December 2014 between the Amy literature model and THM-RR system. The THM-RR measurements ranged between 9.7 and $55.2 \mu \mathrm{g} \cdot \mathrm{L}^{-1}$ Total THMs. Over this same time period the literature model predicted a Total THMs concentration range of 18.6 to $38.6 \mu \mathrm{g} \cdot \mathrm{L}^{-1}$ and the calibrated model predicted 15.4 to $30.6 \mu \mathrm{g} \cdot \mathrm{L}^{-1}$. The Bland-Altman bias for the literature model prediction was $6.3 \pm 5.2 \mu \mathrm{g} \cdot \mathrm{L}^{-1}$, with a $95 \%$ Confidence Interval (CI) of 5.5 to $7.1 \mu \mathrm{g} \cdot \mathrm{L}^{-1}$. In context, the model is predicting concentrations where the MCL for Total THMs is $80 \mu \mathrm{g} \cdot \mathrm{L}^{-1}$. This agreement is excellent for a model applied outside of the water system in which it was developed. The calibrated model performance improved upon this prediction by a factor of three. The Bland-Altman bias for the prediction was $2.0 \pm 4.7 \mu \mathrm{g} \cdot \mathrm{L}^{-1}$ with a $95 \% \mathrm{CI}$ of 1.2 to $2.7 \mu \mathrm{g} \cdot \mathrm{L}^{-1}$. This means that for predictions one calendar year later, the calibrated model predicted Total THMs concentrations to within $\sim 3 \mu \mathrm{g} \cdot \mathrm{L}^{-1}$. As before, the analytical bias provided the same results as the Bland-Altman bias.

The mean $\%$ recovery of the 2014 predictions was $138 \% \pm 33 \%$ for the literature model and $115 \% \pm 22 \%$ for the calibrated model. These are both consistent with the bias measurements. The calibrated model is better at predictions one year later over the literature model, though the mean $\%$ recoveries for both have increased with time compared to the previous year.

As shown in Section 3.3, Bland-Altman plots of Total THMs concentrations generated by the literature and calibrated models $v s$. the THM-RR measurements are presented in Figure 7A,B, respectively. Both show correlation between the model predictions and THM-RR measurements, as expected [39]. The plots of differences are shown in Figure 7C,D, demonstrating no strong trends between the bias and average concentration for both the literature and calibrated models. This means that the error is randomly distributed about the mean and the models have minimal systematic bias in the predictions.

The MUE of the predictive models using both the Amy literature model and the calibrated model were calculated as described earlier by taking the absolute value of each bias and calculating the average. The MUE for the Amy literature model was $6.8 \pm 4.6 \mu \mathrm{g} \cdot \mathrm{L}^{-1}$ while the MUE for the calibrated site specific model was $3.7 \pm 3.4 \mu \mathrm{g} \cdot \mathrm{L}^{-1}$. For the literature model, the difference between the MUE and the Bland-Altman bias is $0.5 \mu \mathrm{g} \cdot \mathrm{L}^{-1}$, and for the calibrated model, $1.7 \mu \mathrm{g} \cdot \mathrm{L}^{-1}$. Both of these differences indicate that the "canceling" effect is minimal and are acceptable values as they are within the error of the bias analysis.

The analyte tracking for the predictive Amy literature and calibrated model showed that the literature model tracked the THMs measurements by the THM-RR system 52\% of the time while the calibrated model tracked the measurements $49 \%$ of the time. The difference in tracking between the two models is negligible. Again, inspection of the two model plots compared to Total THMs measurements by the THM-RR system demonstrates that the calibrated model provides an overall, better visual "match" than the literature model. However, some loss of detail might occur during the calibration process. For example, the seasonal temperature inversion event occurred at the Lebanon, TN WTP between 22 September and 13 October 2013 (Figure 2B). In Figure 4, the Amy literature model exhibits a marked increase in THMs, while the Amy calibrated model does not. A similar example in Figure 6 occurs between 10 October and 18 October 2014; the THM-RR result shows a large increase in THMs. Here, the Amy literature model predicts a larger increase than the Amy calibrated model though neither predict a concentration increase as large as the THM-RR measures. 

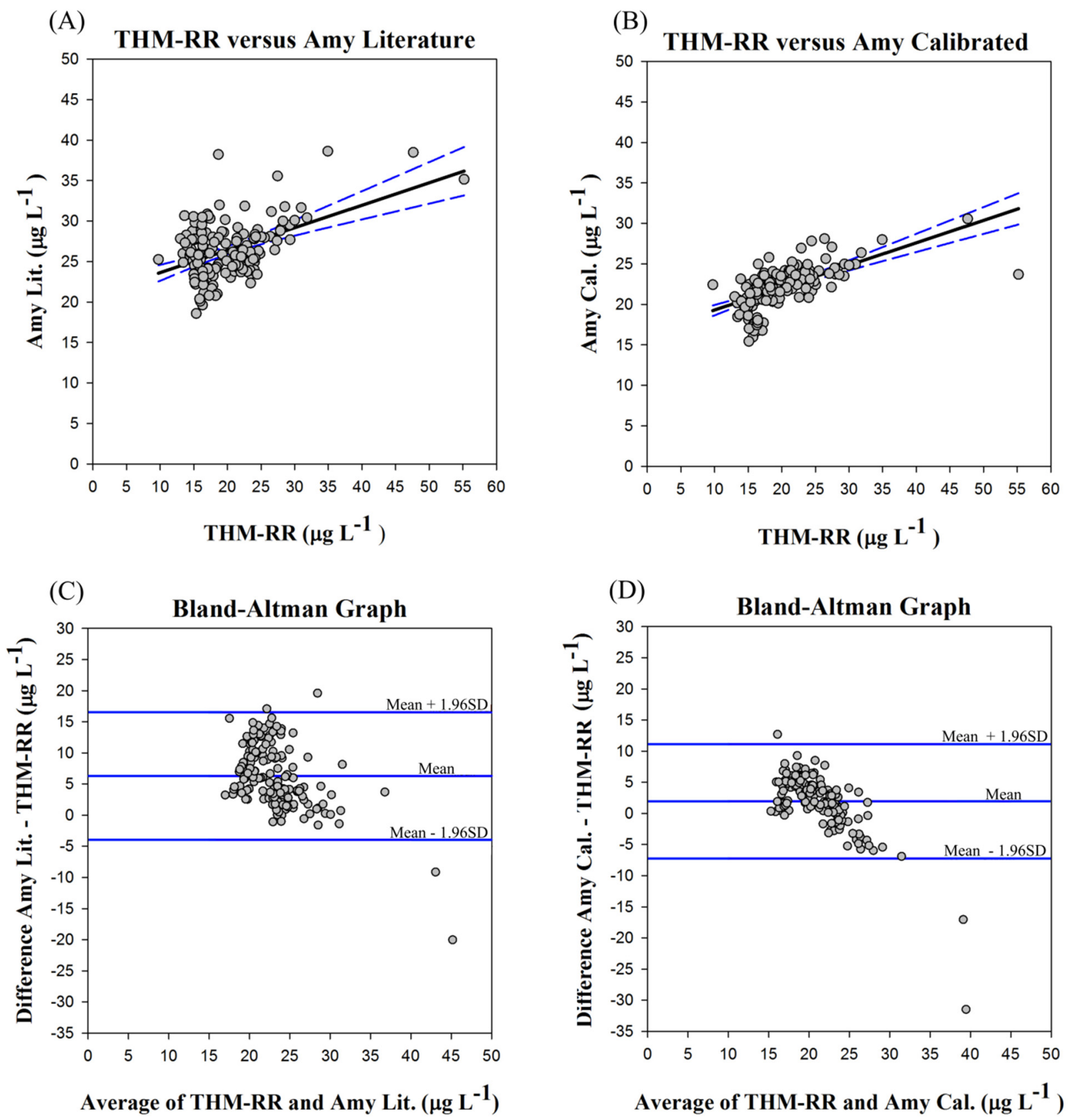

Figure 7. Bland-Altman Plots for the Amy literature and calibrated models for the period of 23 June 2014 through 16 December 2014. (A) Amy Literature generated Total THMs concentrations vs. THM-RR measurements; (B) Amy Calibrated generated Total THMs concentrations vs. THM-RR measurements; (C) Difference plot for the Amy Literature model and THM-RR measurements of Total THMs; (D) Difference plot for the Amy Calibrated model and THM-RR measurements of Total THMs.

\section{Conclusions}

An automated, on-site THMs monitoring program was established at the Lebanon, TN WTP. This was accomplished using the commercially available THM-RR system. The THM-RR was used first to establish a process map that identifies the baseline behavior of the WTP. With the process map in place and baseline behavior understood, process optimization studies were done quantitatively and with 
confidence that observed changes in THMs concentrations were representative of the chemistry occurring during water treatment. Particular attention was paid to optimizing PAC dosage (and thus lowering cost) so that THMs were minimized while improving water quality with respect to THMs occurrence. Further optimization studies were aimed at the pre-chlorination dose to reduce THMs concentrations. Finally, procedures employed by different operators over different shifts were made more uniform. All of these studies combined to yield a WTP that now operates with much less short- and long-term variability in THMs concentrations.

The hourly THMs concentrations measured by the THM-RR were used to evaluate and calibrate a modified version of the well-established Amy empirical model [32]. Bland-Altman analysis was used to evaluate the agreement between the THMs measurements of the THM-RR and the THMs predictions of the empirical model. The modified empirical model, when applied directly from the literature, agreed with THMs measurements from the THM-RR system to an average difference (bias) of about $\operatorname{six~} \mu \mathrm{g} \cdot \mathrm{L}^{-1}$ with error of about five $\mu \mathrm{g} \cdot \mathrm{L}^{-1}$, which is remarkable performance. However, when the model is calibrated by fitting the model results to the THM-RR data, the performance of the model is much improved. The calibrated model was capable of predicting Total THMs concentrations accurately, a full calendar year later, to within $2 \mu \mathrm{g} \cdot \mathrm{L}^{-1}$ with error of about $5 \mu \mathrm{g} \cdot \mathrm{L}^{-1}$.

With the availability of on-site monitoring methods such as the THM-RR system, one might argue that the days of empirical modeling are over. After all, why model when one can now carry out automated on-site monitoring? Nevertheless, the literature is ripe with empirical modeling of THMs concentrations using routine operating water quality data. Such practices are unlikely to end. However, the advent of automated on-site THMs monitoring does provide an opportunity to test the performance of empirical models. These models can be calibrated using on-site THMs monitoring data, thus becoming site specific models optimized for a particular WTP. Combining the practices of on-site monitoring with empirical modeling can lead to two parallel tools for THMs monitoring that operators can use to maintain control and identify potential issues. For example, a WTP monitoring THMs can automatically compare measurements made "on the fly" to calibrated empirical models. Agreement between the measurement and prediction indicate a system operating normally. However, deviation from this agreement, outside of expected norms, indicates either an analytical problem or a treatment process problem. The analytical problem is easily tested using a standard. If there is no analytical problem, the operator can efficiently move toward troubleshooting the problem in the treatment process.

\section{Acknowledgments}

The authors would like to gratefully acknowledge The University of Memphis FedEx Institute of Technology, The University of Memphis Department of Chemistry, Foundation Instruments, Inc., Collierville, TN, USA, and NASA-EPSCoR (Project Number: NNX08AT67A) for portions of this work.

\section{Author Contributions}

Emmert, Simone, and York conceived and designed the experiments; Watts, Brown and Fantom performed the experiments and organized data collection and primary treatment; Snow carried out modeling and agreement measurements. All authors contributed to writing the paper. 


\section{Conflicts of Interest}

As indicated in the credentials section, Emmert is Professor and Chair of the Department of Chemistry at The University of Memphis. He is also a Founder and Chief Scientific Officer of Foundation Instruments, Inc., Collierville, TN, USA, and a shareholder of the company. Simone is an Assistant Professor in the Department of Chemistry at The University of Memphis. He is also a Founder, Director of Research and Development, and shareholder of Foundation Instruments. York and Fantom are employees of the City of Lebanon, TN WTP and responsible for maintaining compliance with water quality regulations. The remaining authors declare no potential conflicts of interest.

\section{References}

1. McGuire, M.J. Eight Revolutions in the History of US Drinking Water Disinfection. J. Am. Water Works Assoc. 2006, 98, 123-149.

2. United States Environmental Protection Agency (USEPA). National Primary Drinking Water Regulations: Disinfectants and Disinfection By-products Rule: Final Rule. Fed. Regist. 1998, 62, 241.

3. USEPA. National Primary Drinking Water Regulations: Stage 2 Disinfectants and Disinfection Byproducts Rule; Final Rule. Fed. Regist. 2006, 71, 388-493.

4. Richardson, S.D.; Plewa, M.J.; Wagner, E.D.; Schoeny, R.; DeMarini, D.M. Occurrence, genotoxicity, and carcinogenicity of regulated and emerging disinfection by-products in drinking water: A review and roadmap for research. Mutat. Res. 2007, 636, 178-242.

5. Bond, T.; Goslan, E.H.; Parsons, S.A.; Jefferson, B. A critical review of trihalomethanes and haloacetic acid formation from natural organic matter surrogates. Environ. Tech. Rev. 2012, 1, 93-113.

6. Environmental Working Group. National Drinking Water Database. Available online: http://www.ewg.org/tap-water/ (accessed on 26 May 2015).

7. Brown, D.; Bridgeman, J.; West, J.R. Understanding data requirements for trihalomethanes formation modelling in water supply systems. Urban Water J. 2011, 8, 41-56.

8. Doederer, K.; Gernjak, W.; Weinberg, H.S.; Farré, M.J. Factors affecting the formation of disinfection by-products during chlorination and chloramination of secondary effluent for the production of high quality recycled water. Water Res. 2014, 48, 218-228.

9. Chowdhury, S.; Rodriguez, M.J.; Serodes, J. Model development for predicting changes in DBP exposure concentrations during indoor handling of tap water. Sci. Total Environ. 2010, 408, 4733-4743.

10. Mosteo, R.; Miguel, N.; Martin-Muniesa, S.; Ormad, M.P.; Ovelleiro, J.L. Evaluation of trihalomethanes formation potential in function of oxidation processes used during the drinking water production process. J. Hazard. Mater. 2009, 172, 661-666. 
11. Weinberg, H.S.; Krasner, S.W.; Richardson, S.D.; Thruston, A.D., Jr. The Occurrence of Disinfection by-Products (DBPs) of Health Concern in Drinking Water: Results of a Nation-Wide DBP Occurrence Study; EPA/600/R-02/068; EPA: Athens, GA, USA, 2002. Available online: www.epa.gov/Athens/publications/reports/EPA_600_R02_068.pdf (accessed on 28 May 2015).

12. Edzwald, J.K.; Becker, W.C.; Wattier, K.L. Surrogate Parameters for monitoring organic matter and THM precursors. J. Am. Water Works Assoc. 1985, 77, 122-132.

13. Brown, Aaron W.; Simone, P.S., Jr.; York, J.C.; Emmert, G.L. A device for fully automated on-site process monitoring and control of trihalomethane concentrations in drinking water. Anal. Chim. Acta 2015, 853, 351-359.

14. Liang, L.; Singer, P.C. Factors Influencing the Formation and Relative Distribution of Haloacetic Acids and Trihalomethanes in Drinking Water. Environ. Sci. Technol. 2003, 37, 2920-2928.

15. Consonery, P.J.; Lusardi, P.J.; Kopansky, R.; Manning, R.L. Total Organic Carbon: A Reliable Indicator of TTHM and HAA5 Formation? In Proceedings of the Water Quality Technology Conference, San Antonio, TX, USA, 14-17 November 2004.

16. Emmert, G.L.; Cao, G.; Geme, G.; Joshi, N.; Rahman, M. Methods for Real-Time Measurement of Trihalomethanes and Haloacetic Acids in Distribution Systems; Final Report; American Water Works Research Foundation: Denver, CO, USA, 2004.

17. USEPA. Method 551.1. Determination of Chlorination Disinfection by-Products, Chlorinated Solvents, and Halogenated Pesticides/Herbicides in Drinking Water by Liquid-Liquid Extraction and Gas Chromatography with Electron-Capture Detection; Environmental Monitoring and System Laboratory: Cincinnati, OH, USA, 1998.

18. USEPA. Method 502.2. Volatile Organic Compounds in Water by Purge and Trap Capillary Gas Chromatography with a Photoionization and Electrolytic Conductivity Detector in Series; National Exposure Research Laboratory Office of Research and Development US Environmental Protection Agency: Cincinnati, OH, USA, 1995.

19. USEPA. Method 524.2. Measurement of Purgeable Organic Compounds in Water by Capillary Column Gas Chromatography/Mass Spectrometry; Environmental Monitoring and System Laboratory: Cincinnati, OH, USA, 1995.

20. Hach THM II Plus. Total Trihalomethanes (THM) Reagent Set. Available online: http://www.hach.com/total-trihalomethanes-thm-reagent-set/product?id=7640197853 (accessed on 1 October 2015).

21. Parker Hannifin. The Parker THM Analyzer. Available online: http://www.parker.com/ literature/Instrumentation\%20Products\%20Division/Catalogs/WATER_THM_Bulletin.pdf (accessed on 2 October 2015).

22. Multisensor Systems. Total Trihalomethanes (THM) Monitor. 2000. Available online: http://www.multisensor.co.uk/product.asp/id/2/THM-monitor.htm (accessed on 1 October 2015).

23. Aqua Metrology Systems. THM-100. 2012. Available online: http://www.aquametrologysystems.com/thm-100/ (accessed on 1 October 2015).

24. Emmert, G.L.; Brown, M.A. Real-Time, on-Line Analysis for the Quantification of Trihalomethane Species within Drinking Water Supplies. U.S. Patent 8,336,371, 25 December 2012. 
25. Emmert, G.L.; Brown A.W.; York J.C.; Simone, P.S., Jr. Establishing an automated online monitoring program for trihalomethane control at the Lebanon, Tennessee Water Treatment Plant. Straight from the Tap, winter 2014, pp. 34-38.

26. Brown, M.A.; Emmert, G.L. On-line monitoring of trihalomethane concentrations in drinking water distribution systems using capillary membrane sampling-gas chromatography. Anal. Chim. Acta 2006, 555, 75-83.

27. Emmert, G.L.; Brown, M.A.; Simone, P.; Cao, G. Methods for Real-time Measurement of Trihalomethanes and Haloacetic acids in Distribution Systems; American Water Works Research Foundation: Denver, CO, USA, 2007.

28. Rook, J.J. Chlorination reactions of fulvic acids in natural waters. Environ. Sci. Technol. 1977, 11, 478-482.

29. Minear, R.; Morrow, C. Raw Water Bromide: Levels and Relationship to Distribution of Trihalomethanes in Finished Drinking Water; Research Report No. 91; Water Resources Research Center, University of Tennessee: Knoxville, TN, USA, 1983.

30. Chowdhury, S.; Champagne, P.; McLellan, P.J. Models for predicting disinfection byproduct (DBP) formation in drinking waters: A chronological review. Sci. Total Environ. 2009, 407, 4189-4206.

31. Amy, G.L.; Chadik, P.A.; Chowdhury, Z.K. Developing Models for Predicting Trihalomethane Formation Potential and Kinetics. J. Am. Water Works Assoc. 1987, 79, 89-97.

32. Amy, G.L.; Siddiqui, M.; Ozekin, K.; Zhu, H.W.; Wang, C. Empirically Based Models for Predicting Chlorination and Ozonation by-Products: Trihalomethanes, Haloacetic Acids, Chloral Hydrate, and Bromate; USEPA 1998 EPA report CX 819579; USEPA: Cincinnati, OH, USA, 1998.

33. Glaser, J.A.; Forest, D.L. Trace Analysis of Wastewaters. Environ. Sci. Technol. 1981, 15, $1426-1435$.

34. USEPA. Appendix B, Part 136-Definition and Procedure for the Determination of the Method Detection Limit-Revision 1.11. Fed. Regist. 1996, 71, 303-306.

35. Skoog, D.A.; Holler, F.H.; Nieman, T.A. Principles of Instrumental Analysis, 5th ed.; Harcourt Brace \& Company: San Diego, CA, USA, 1998.

36. USEPA. DBP/ICR Analytical Methods Manual; USEPA: Cincinnati, OH, USA, 1996.

37. Systat Software Inc. (San Jose, CA, USA). SigmaPlot Version 13.0. Available online: http://www.sigmaplot.com (accessed on 26 May 2015).

38. Motulsky, H.J.; Ransnas, L.A. Fitting curves to data using nonlinear regression: A practical and nonmathematical review. FASEB J. 1987, 1, 365-374.

39. Altman, D.G.; Bland, J.M. Measurement in Medicine: The Analysis of Method Comparison Studies. Statistician 1983, 32, 307-317.

40. Bland, J.M.; Altman, D.G. Statistical methods for assessing agreement between two methods of clinical measurement. Lancet 1986, 327, 307-310.

41. Bland, J.M.; Altman, D.G. Comparing methods of measurement: Why plotting difference against standard method is misleading. Lancet 1995, 346, 1085-1087.

42. Bland, J.M.; Altman, D.G. Measuring agreement in method comparison studies. Stat. Methods Med. Res. 1999, 8, 135-160. 
43. Samarasinghe, S. Neural Networks for Time-Series Forecasting. Neural Networks for Applied Sciences and Engineering: From Fundamentals to Complex Pattern Recognition; Auerbach: Boca Raton, FL, USA, 2007; pp. 460-462.

(C) 2015 by the authors; licensee MDPI, Basel, Switzerland. This article is an open access article distributed under the terms and conditions of the Creative Commons Attribution license (http://creativecommons.org/licenses/by/3.0/). 